\title{
Continuous Dynamic Recrystallization During Severe Plastic Deformation
}

\author{
Mattia Bacca ${ }^{1,2, *}$, David R. Hayhurst ${ }^{3}$, Robert M. McMeeking ${ }^{1,2,45}$ \\ ${ }^{1}$ Mechanical Engineering Department, University of California, Santa Barbara CA 93106, USA \\ ${ }^{2}$ Materials Department, University of California, Santa Barbara CA 93106, USA \\ ${ }^{3}$ Mechanical, Aerospace and Civil Engineering, University of Manchester, Manchester M60 \\ 1QD, UK \\ ${ }^{4}$ School of Engineering, University of Aberdeen, King's College, Aberdeen AB24 3UE, UK \\ ${ }^{5}$ INM - Leibniz Institute for New Materials, Campus D2 2, 66123 Saarbrücken, Germany \\ *Corresponding author: mattia.bacca@engineering.ucsb.edu
}

\begin{abstract}
Severe plastic deformation (strains $>100 \%$ ) has been shown to create significant grain refinement in polycrystalline materials, leading to a nanometric equiaxed crystalline structure for such metals as aluminum, copper and nickel alloys. This process, termed continuous dynamic recrystallization, is governed by evolution of the dislocation structure, which creates new grain boundaries from dislocation walls. In the proposed model, plasticity occurs which firstly involves dislocation multiplication, leading to strain hardening limited by dynamic recovery. After a critical dislocation density is reached new grain boundaries are formed by condensation of walls of dislocations, creating a new stable configuration that is favored due to a reduction of the system free energy. This evolution of the microstructure continues to develop, with a consequent progressive decrease in the average grain diameter. The proposed model provides a quantitative prediction of the evolution of the average grain size, as well as the dislocation density, during continued plastic strain. The model can be calibrated by use of results from any experiment that involves large plastic deformation of metals, subject to negligible annealing effects. In this paper, the model has been calibrated, and consequently validated, through experiments on machining of Al 6061-T6.
\end{abstract}

Keywords: microstructure, evolution, continuous dynamic recrystallization, machining

\section{Introduction}

Grain refinement is an important feature in many thermo-mechanical processes involved in manufacture engineering, producing material that is much stronger than that without grain refinement. This phenomenon can be achieved in several ways, the most common one being the activation of recrystallization (RX) by severe plastic deformation (SPD), with a consequent decrease in the average diameter of the grains. Severe plastic deformation typically involves plastic strains greater than 1 in manufacturing processes such as machining or metal cutting [1] and equal channel angular pressing (ECAP) [2,3], the former being the most effective since the maximum amount of shear equivalent plastic strain can be up to 15 [1]. This processes are able to create grain sizes in the range of nanometers, and such nanostructured materials are known to have much higher strength than those having grain size on the micron scale. Grain boundaries act as barriers to dislocation motion, with pile-ups forming whose extent is constrained by the grain size so that the driving force for them to penetrate the barrier is limited, i.e. the Hall-Petch effect [4-5]. As a consequence the yield strength of the material is inversely proportional to the square 
root of grain size, leading to significant strengthening in nanostructured materials. The driving force for RX is the energy stored in the network of dislocations that is generated during plastic deformation. A fraction of the plastic work associated with such deformation is stored in this dislocation network. Once this energy becomes excessive the system finds it thermodynamically more favorable to reduce its free energy by condensing the dislocation structure into new grain boundaries associated with small, lower dislocation density crystallites.

A thermodynamically consistent model for RX during SPD, inspired by Le and Kochmann [6], is proposed in this paper; it is described in Section 2, its calibration is reported in Section 3, computational results derived from the model are reported in Section 4 and compared with experiments on machining for the Aluminium Alloy Al 6061-T6 [1], a discussion on the model and its comparison with other models is reported in Section 5 and its implementation in a finite element code is reported in Section 6.

\section{Proposed Model}

The mechanical properties of a material are defined by its microstructure, and plastic deformation in metals affects these properties by modifying microstructural internal variables that control them [7]. For example, a common model for yield strength in a metal superposes Taylor strengthening by dislocation-dislocation pinning [8] and the Hall-Petch effect [4-5] due to dislocation pile-ups interacting with grain boundaries to give the yield strength in shear, $\tau_{y}$, as [9]

$\tau_{y}=\alpha b \mu \sqrt{\rho}+\lambda\left(\frac{1}{\sqrt{d}}-\frac{1}{\sqrt{d_{0}}}\right)$

where $\alpha$ is a phenomenological constant for Taylor hardening (usually taken as unity), $b$ is the Burgers vector, $\mu$ is the shear modulus, $\rho$ is the dislocation density $\lambda$ is the Hall-Petch coefficient, $d$ is the current average grain size, and $d_{0}$ is a reference grain size that is sufficiently large so that the Hall-Petch effect is negligible at that grain size. Plastic deformation causes the dislocation density to increase by dislocation multiplication at sources in the material and, as noted above, can also cause the grain size to change due to RX. The proposed model, based on a simple one developed by Le and Kochmann [6] for continuous dynamic recrystallization (CDRX), describes the evolution of the dislocation density and the grain size on a thermodynamically consistent basis, as follows.

Consider the energy conservation during plastic deformation, but neglect the elastic strain energy of the macroscopic deformation as it is stored reversibly (see [6] for more details). Thus only dissipative terms are included, giving

$\tau_{y} \dot{\gamma}_{p}=\dot{Q}+\dot{U}_{m}$

where $\dot{\gamma}_{p}$ is the rate of change of the shear equivalent plastic strain, $\dot{Q}$ is the rate per unit volume at which mechanical work is dissipated as heat, and $\dot{U}_{m}$ is the rate per unit volume at 
which plastic work is stored in the microstructure (the latent heat rate) [10]. As is well known, the left hand side of eq. (2) is the rate per unit volume at which the applied loads do provide plastic work to the material. Defining now a latent heat capacity as the fraction, $\kappa$, of plastic work being stored in the microstructure, then,

$\dot{U}_{m}=\kappa \tau_{y} \dot{\gamma}_{p}$.

At this point it is useful to describe the microstructural energy, $U_{m}$, as estimated by Berdichevsky [11] as a function of the two microstructural parameters, such that

$U_{m}(\rho, \mathrm{d})=-k \mu\left[\log \left(1-\sqrt{\frac{\rho}{\rho_{s}}}\right)+\sqrt{\frac{\rho}{\rho_{s}}}\right]+\frac{\chi \Gamma_{s}}{2 d}$,

where $k$ is a constant, $\chi$ is a grain shape factor (its value is 3 if we consider spherical grains), $\Gamma_{s}$ is the surface energy of the grain boundary, and $\rho_{s}$ is a theoretical saturation limit for the dislocation density; i.e. in the absence of $\mathrm{RX} \rho_{s}$ is the maximum dislocation storage capacity. By substitution of eq. (1) and eq. (4) into eq. (3) a differential equation in the rate of change of the two main variables $\rho$ and $d$ is obtained

$$
\frac{k \mu}{2 \rho_{s}\left(1-\sqrt{\rho / \rho_{s}}\right)} \dot{\rho}-\frac{\chi \Gamma_{s}}{2 d^{2}} \dot{d}=\kappa\left[\alpha b \mu \sqrt{\rho}+\lambda\left(\frac{1}{\sqrt{d}}-\frac{1}{\sqrt{d_{0}}}\right)\right] \dot{\gamma}_{p} .
$$

As many theories and experiments indicate, RX commences only after a critical amount of plastic deformation is reached, associated with instability in the intragranular dislocation structure [12-14]. This instability leads to the creation of low angle subgrain boundaries condensed from existing dislocations in the microstructure. At this point RX has started and all existing dislocations and many of the new ones produced will eventually be adsorbed into the subgrain boundaries, causing increasing misorientation across them until new, distinct, refined grains are created.

From now on, microstructural evolution is divided into two main stages: prior to Recrystallization, and during Recrystallization (equivalent to Stage I and Stage II respectively in the model proposed by Le and Kochmann [6]).

\subsection{Prior to Recrystallization}

Before RX starts, grain size is approximately uniform and constant, equal to the initial value $d=d_{0}$ [6]. Experiments show that in this stage the biggest contribution to strain hardening is due to the continuous increase of dislocation density due to their multiplication at sources [14, 15]. In this situation the evolution of the dislocation density, $\rho$, is associated with two competing processes; their multiplication in Frank-Read sources, and dynamic recovery due to dislocation-dislocation annihilation, as described by Kocks [16] in the formula, 
$\frac{\partial \rho}{\partial \gamma_{p}}=k_{1} \sqrt{\rho}-k_{2}(\dot{\gamma}, T) \rho$

In eq. (6) the constant $k_{1}$ describes the Frank-Read dislocation loop process and leads to an increase in the dislocation density, while $k_{2}(\dot{\gamma}, T)$ describes dynamic recovery, and leads to a decrease in $\rho$. As indicated, $k_{2}$ depends on the shear equivalent strain rate, $\dot{\gamma}, 1$, and on $T$, the temperature. The solution for eq. (6) that Kocks [16] provided, for constant strain rate and temperature, is

$\sqrt{\rho\left(\gamma_{p}, \dot{\gamma}, T\right)}=\sqrt{\rho_{0}}+\left[\sqrt{\rho_{s}(\dot{\gamma}, T)}-\sqrt{\rho_{0}}\right]\left\{1-\exp \left[-\gamma_{p} / \gamma_{0}(\dot{\gamma}, T)\right]\right\}$,

where, $\sqrt{\rho_{s}(\dot{\gamma}, T)}=\sqrt{\rho_{0}}+\left(\Delta \tau_{y, s 0} / \alpha \mathrm{b} \mu\right) f(\dot{\gamma}, T), \gamma_{0}(\dot{\gamma}, T)=\bar{\gamma}_{0} f(\dot{\gamma}, T), \rho_{0}$ is the initial dislocation density and $\Delta \tau_{y, 50}$ is a representative yield strength. The function $f(\dot{\gamma}, T)=\left(\dot{\gamma} / \dot{\gamma}_{0}\right)^{-\bar{k} T / A^{\prime}}$ keeps track of the conditions of the experiment, $\dot{\gamma}_{0}$ is a reference strain rate, $\bar{k}$ is Boltzmann's constant, and $A^{\prime}$ is the activation energy for dynamic recovery. Equation (5) following substitution of eq. (7), for $d=d_{0}$ and $\dot{d}=0$, becomes,

$k(\dot{\gamma}, T)=\kappa \alpha b \sqrt{\rho_{s}(\dot{\gamma}, T)} \gamma_{0}(\dot{\gamma}, T)$

After substitution of eq. (7) into eq. (1), for $d=d_{0}$, one finally obtain a formulation for the strain hardening that has the same form as the Voce law [17],

$\tau_{y}\left(\gamma_{p}, \dot{\gamma}, T\right)=\tau_{y, 0}+\left[\tau_{y, s}(\dot{\gamma}, T)-\tau_{y, 0}\right]\left\{1-\exp \left[-\gamma_{p} / \gamma_{0}(\dot{\gamma}, T)\right]\right\}$,

with $\tau_{y, s}(\dot{\gamma}, T)=\tau_{y, 0}+\Delta \tau_{y, s 0} f(\dot{\gamma}, T)$. On definition of a hardening parameter as $\theta=\partial \tau_{y}\left(\gamma_{p}, \dot{\gamma}, T\right) / \partial \gamma_{p}$, its initial value can be expressed as

$\theta_{0}=\left.\frac{\partial \tau_{y}\left(\gamma_{p}, \dot{\gamma}, T\right)}{\partial \gamma_{p}}\right|_{\gamma_{p}=0}=\frac{\Delta \tau_{y, s, 0}}{\bar{\gamma}_{0}}$,

which is independent of temperature and strain rate. For this reason $\theta_{0}$ is considered to be a material parameter [16].

\footnotetext{
${ }^{1}$ In the context of SPD, as a first approximation, elastic strains are not considered so that strain rate is equal to plastic strain rate.
} 


\subsubsection{Measure of the Latent Heat Capacity}

The first term on the right hand side of eq. (4) shows the relationship between latent heat $U_{m}$ and dislocation density $\rho$. For very small dislocation density the former becomes a linear function of the latter, since the lattice distortion energy, for a unit line dislocation, is $\mu b^{2}$, so that

$\left.U_{m}(\rho)\right|_{\rho \rightarrow 0}=\mu b^{2} \rho$.

Linearization of eq. (4) with respect to $\rho$, comparison with eq. (11), and substitution of eq. (10) and eq. (8) into it, gives,

$$
\kappa=\frac{2 \theta_{0}}{\alpha^{2} \mu} .
$$

From eq. (12) one can derive the latent heat capacity as a material parameter. Considering $\alpha=1$ [6] and $\theta_{0}=0.06 \mu$ [16], we obtain $\kappa=12 \%$, which is close to the value used by Le and Kochmann [6].

\subsection{During Recrystallization}

Dislocation density accumulates until a critical level $\rho=\rho_{c}$ is reached, and RX starts. As a consequence a critical amount of plastic strain, necessary to obtain $\rho_{c}$, initiates RX. Some theories exist for the evaluation of $\rho_{c}$ [12-13]; in the proposed model, the value arises from a condition of instability leading to the RX process, associated with the maximum value of $\rho$ for which the condition $\dot{U}_{m}>0$ holds. This is in agreement with the hypothesis of Le and Kochmann [6]; its evaluation is reported in paragraph 3.2, below.

A steady state in the yield strength is commonly assumed during continuous dynamic recrystallization CDRX e.g. [6,12], finding good agreement with experiments [14-15]. This means that the strain hardening due to dislocation multiplication is balanced by a combination of dynamic recovery and dislocation adsorption by generation of new grains. This hypothesis can be written,

$\tau_{y}=\tau_{y, \mathrm{ss}}, \dot{\tau}_{y}=0$.

where $\tau_{y, s s}$ is the steady state value. From eq. (13) and eq. (1), the following can be written,

$$
\sqrt{\rho}+\frac{\lambda}{\alpha b \mu}\left(\frac{1}{\sqrt{d}}-\frac{1}{\sqrt{d_{0}}}\right)=\sqrt{\rho_{c}} \text {, and } \frac{\dot{\rho}}{\sqrt{\rho}}=\frac{\lambda}{\alpha b \mu} \frac{\dot{d}}{d \sqrt{d}} \text {. }
$$

Eq. (14) and eq. (8) can be substituted into eq. (5) to give the differential equation, 


$$
\frac{1}{2}\left[\frac{\kappa \gamma_{0} \lambda}{d \sqrt{d}} \frac{\sqrt{\frac{\rho_{c}}{\rho_{s}}}-\frac{\lambda}{\tau_{y, s}}\left(\frac{1}{\sqrt{d}}-\frac{1}{\sqrt{d_{0}}}\right)}{1-\sqrt{\frac{\rho_{c}}{\rho_{s}}}+\frac{\lambda}{\tau_{y, s}}\left(\frac{1}{\sqrt{d}}-\frac{1}{\sqrt{d_{0}}}\right)}-\frac{\chi \Gamma_{s}}{d^{2}}\right] \dot{d}=\kappa\left[\alpha b \mu \sqrt{\rho}+\lambda\left(\frac{1}{\sqrt{d}}-\frac{1}{\sqrt{d_{0}}}\right)\right] \dot{\gamma}_{p},
$$

whose solution gives a relationship between grain size $d$ and plastic strain $\gamma_{p}$. The critical dislocation density $\rho_{c}$ at which RX commences is undetermined at this stage, but is discussed in Section 3.3 in conjunction with calibration of the model.

\section{Calibration of the model}

To use the model for the Aluminum alloy Al 6061-T6 the material parameters are given in Table 1 , together with their sources.

\begin{tabular}{|l|ll|}
\hline Parameter & Value for Al 6061-T6 & \\
\hline$\mu$ & $25 \mathrm{GPa}$ & {$[6]$} \\
$\chi$ & 3 & {$[6]$} \\
$\gamma_{s}$ & $0.625 \mathrm{~J} / \mathrm{m}^{2}$ & {$[6]$} \\
$\lambda$ & $0.01 \mathrm{MPa} \cdot \mathrm{m}^{1 / 2}$ & {$[6]$} \\
$\alpha$ & 1 & {$[8]$} \\
$b$ & $4 \cdot 10^{-10} \mathrm{~m}$ & {$[6]$} \\
$d_{0}$ & $75 \mu \mathrm{m}$ & {$[1]$} \\
$\theta_{0} / \mu$ & 0.06 & {$[16]$} \\
\hline
\end{tabular}

Table 1: Material parameters for Al 6061-T6.

\subsection{Description of the machining experiment}

The proposed model has been calibrated for a machining experiment on the Aluminum alloy $\mathrm{Al}$ 6061-T6 performed by Shankar et. al. [1]. The test consists in material removal with a cutting tool that has the cutting surface oriented with a specific rake angle $\vartheta$ with respect to its trajectory. The depth of removed material $a_{0}$ is called the feed and it corresponds to the initial thickness of the chip $a$ before it is removed. The removal is done by advancement of the tool at a controlled cutting speed $v_{0}$ at room temperature. Frictional heat generation at the interface between the cutting tool and the chip is expected with a consequent increase in the temperature of the specimen within the chip and on the machined surface. For a sufficiently low cutting speed, as measured in the referenced experiments [1], the temperature rise is negligible.

In Figure 1 a schematic representation of the experiment is shown and in Table 2 rake angle, feed and cutting speed of the experiment considered [1] are reported. In this table, shear plastic strain produced inside the chip is also reported as it can be estimated from $\vartheta$ and the measure of $a$; three values are shown as three different rake angles have been used to obtain 3 sets of results. In Figure 1, the primary deformation zone (PDZ) is shown to extend from the edge of the cutting 
tool along the foot of the chip, and represents the boundary between the un-deformed material being removed and the deformed material inside the chip.

\begin{tabular}{|l|l|c|}
\hline Parameter & Symbol & Values from [1] \\
\hline Rake angle & $\vartheta$ & $20^{\circ},-5^{\circ},-20^{\circ}$ \\
Shear plastic strain & $\gamma_{p}$ & $1.7, \quad 3.2,5.2$ \\
Feed & $a_{0}$ & $0.150 \mathrm{~mm}$ \\
Cutting speed & $v_{0}$ & $10 \mathrm{~mm} / \mathrm{s}$ \\
\hline
\end{tabular}

Table 2: Rake angle, shear plastic strain, feed and cutting speed for the experiments on machining by Shankar et al [1].

\subsection{Calibration on Vicker Hardness}

From experiments on machining for Al 6061-T6 carried out by Shankar et al. [1], evolution of Vickers hardness $H_{v}$ with respect to the plastic strain $\gamma_{p}$ has been measured and reported as in Figure 2. A linear relationship between Vickers hardness and yield strength is well established and given by

$$
\sigma_{y}=K H_{v}
$$

with $K \approx 0.3$ [18]. Therefore from Figure 2 the evolution of the yield shear strength with plastic shear strain can be obtained. It is hypothesized that RX initiates at small plastic strain, so that it can be assumed that a steady state condition exists for the hardness values (and consequently for the yield strength) for all experimental data points shown in Fig. 2, other than that at zero plastic strain when the hardness of the material is that prior to machining.

A value for the steady state Hardness $H_{v, s s}$ has been determined from the data on a least squares fit, while the hardness reported in [1] has been taken for the initial value $H_{v, o}$, leading to the values

$$
H_{v, 0}=110 \mathrm{~kg} / \mathrm{mm}^{2}, H_{v, s s}=152 \mathrm{~kg} / \mathrm{mm}^{2} .
$$

From eq. (16) we have,

$$
\begin{aligned}
& \sigma_{y, 0} \approx 0.3 \cdot 110=33 \mathrm{Kg} / \mathrm{mm}^{2}=323.73 \mathrm{MPa}, \\
& \sigma_{y, s s} \approx 0.3 \cdot 152=45.6 \mathrm{Kg} / \mathrm{mm}^{2}=447.336 \mathrm{MPa},
\end{aligned}
$$

and thus

$$
\tau_{y, 0}=\frac{\sigma_{y, 0}}{\sqrt{3}}=186.91 \mathrm{MPa}, \tau_{y, s s}=\frac{\sigma_{y, s s}}{\sqrt{3}}=258.27 \mathrm{MPa}
$$


The hypothesis of steady state hardness allows it to be fitted to the data in Figure 2 in such a way that at least one of the extremities of each error bar is within $2 \%$ of the estimated hardness. In the experiments on machining [1], a low cutting speed of $10 \mathrm{~mm} / \mathrm{s}$ was adopted in order to minimize the influence of the shear strain rate $\dot{\gamma}$ and the temperature $T$ in the mechanical behavior of the specimen. This condition further validates the hypothesis of steady state behavior in the yield strength in all the experiments shown in Figure 2 since the influence of strain rate and temperature is negligible, and thus all the points shown belong to the same stress-strain curve for materials all in the same condition ${ }^{2}$.

\subsection{Critical condition for initiation of Recrystallization}

$\mathrm{RX}$ starts after a critical density of dislocations is reached that constitutes an unstable configuration, giving rise to nucleation of new grain boundaries. However, during continuing RX (i.e. CDRX) plastic work is partly dissipated by heat, but a positive fraction of the plastic work is stored in the microstructure [6]. Since the right hand side of eq. (15) is this fraction of the plastic work, given the current model it must remain positive and finite. Our physical picture is that upon initiation of RX there is a burst of it associated with the instability of the dislocation network, and the rate of change of grain size becomes equal to $-\infty$. In this situation the only way to keep the left hand side of eq. (15) positive and finite is if the coefficient of $\dot{d}$ goes to zero. We take this to be the signature of the instability of the dislocation network initiating RX. This condition, at RX initiation (where $d=d_{0}$ ), gives the following equality,

$\frac{\sqrt{\rho_{c} / \rho_{s}}}{1-\sqrt{\rho_{c} / \rho_{s}}}-\frac{\chi \Gamma_{s}}{\kappa \lambda \gamma_{0} \sqrt{d_{0}}}=0$

From eq. (19) one obtains,

$\sqrt{\frac{\rho_{c}}{\rho_{s}}}=\frac{1}{1+\varphi \kappa \gamma_{0}}$,

where $\varphi=\lambda \sqrt{d_{0}} /\left(\chi \Gamma_{s}\right)$. Given that $\tau_{y, c}=\tau_{y, s s}, \sqrt{\rho_{c} / \rho_{s}}=\tau_{y, s s} / \tau_{y, s} \quad$ and $\gamma_{0}=\left(\tau_{y, s}-\tau_{y, 0}\right) / \theta_{0}=\left(\tau_{y, s s} \sqrt{\rho_{s} / \rho_{c}}-\tau_{y, 0}\right) / \theta_{0}$, it is finally deduced that

$\sqrt{\frac{\rho_{c}}{\rho_{s}}}=\frac{1-\varphi \kappa \tau_{y, s s} / \theta_{0}}{1-\varphi \kappa \tau_{y, 0} / \theta_{0}}$

When these parameters are calibrated it is important to ensure that the algorithm arising from the equations operates on the correct branch of the response, thereby causing the grain size to diminish and not increase. This condition can be assured by selecting a value for $\rho_{c}$ slightly below the result in eq. (21).

\footnotetext{
${ }^{2}$ In general, different rake angles can give different strain rates with the same cutting speed.
} 
Considering the values for the physical parameters of aluminum alloy Al6061-T6 reported in Table 1, one obtains $\varphi=46.18802154$, which gives $\sqrt{\rho_{c} / \rho_{s}}=0.147664891$. Truncating these values at the third digit, one finds a final value as $\sqrt{\rho_{c} / \rho_{s}}=0.147$, which we find to be low enough to keep the algorithm on the correct branch of the solution, with grain size diminishing. The square root of the ratio of the initial dislocation density to the saturation value is then $\sqrt{\rho_{0} / \rho_{s}}=\sqrt{\rho_{c} / \rho_{s}} \tau_{y, 0} / \tau_{y, s s}=0.106$. From this $\gamma_{0}=1.05$ can be obtained and then the critical shear strain for RX initiation can be determined from eq. (7) as,

$$
\gamma_{c}=\gamma_{0} \log \left(\frac{1-\sqrt{\rho_{0} / \rho_{s}}}{1-\sqrt{\rho_{c} / \rho_{s}}}\right)=0.0493
$$

Note that our values for $\sqrt{\rho_{0} / \rho_{s}}, \gamma_{0}$ and $\gamma_{c}$ are rounded to 3 significant figures. Given our truncation of $\sqrt{\rho_{c} / \rho_{s}}$ to 3 figures, we find that rounding $\sqrt{\rho_{0} / \rho_{s}}, \gamma_{0}$ and $\gamma_{c}$ to 2 significant figures may destabilize the algorithm, placing it on the wrong solution branch, and causing the grain size to grow. Therefore, given truncation of $\sqrt{\rho_{c} / \rho_{s}}$ to 3 figures, rounding of $\sqrt{\rho_{0} / \rho_{s}}$, $\gamma_{0}$ and $\gamma_{c}$ to 3 significant figures or greater should be used and rounding to less than 3 avoided.

Note that the value of $\gamma_{c}$ from Eq. (22) is well below the strain value for all the data points in Figure 2. This observation is consistent with the fact that RX has occurred for all these data.

\section{Results}

After the calibration of the model, the plot of eq. (15) for grain size prediction is inferred, and a comparison of this result with experimental data from machining is reported in Figure 3. Experimental results are reported in this figure with three circles and relative error bars showing a tolerance of $50 \%$ (this is an estimation of the accuracy of the geometrical technique used for measuring the average grain size from transmitted electron microscopy (TEM) images). The left figure shows a logarithmic plot while the right figure shows an arithmetic plot, and both evidence a qualitative matching to an order of magnitude at the nanometer scale. The arithmetic plot also shows good matching in the slope of the curve, especially between the two left experimental points. The horizontal asymptote exhibited by the experiments, at around $80 \mathrm{~nm}$ as evidenced in the two right experimental points (at $d / d_{0} \approx 10^{-3}$, from Table 1 ), is not captured by the model, which tends to give a continual decrease in the grain size throughout the entire domain reported in the figure.

The evolution of the dislocation density is reported in Figure 4, where a continual decrease can be seen. In this figure, the dislocation density is normalized by the saturation density $\rho_{s}$; the initial ratio is $\rho_{c} / \rho_{s}=(0.147)^{2}=0.0216$, and the ratio decreases to zero for very large plastic strains. When zero dislocation density is reached, it is not possible to generate new grain boundaries, therefore microstructural evolution is completed and other deformation mechanisms have to come into play. 
As a first approximation, all the material parameters have been considered constant during the whole process of recrystallization. The reason for this assumption relies on the shorter time frame in which the process occurs, being based on dislocation glide, with respect to phenomena such as temperature rise by frictional heat generation (for the case of machining) etc. After recrystallization is complete, annealing effects are expected as a consequence of heat generations, creating coarsening of the grain structure via grain growth. In alloys such $\mathrm{Al} \mathrm{6061-}$ T6, precipitates of the secondary phases tend to pin the grain boundaries and prevent them from moving, therefore preserving the refined grain structure.

\section{Discussion}

The proposed model gives a good prediction of the evolution of the average grain size as evidenced in Figure 3, it is much simpler than other proposed models [19, 20], having fewer physical parameters, and it can be calibrated easily for any large plastic strain experiment for any polycrystalline metal.

As stated at the beginning of this paper, the proposed model is inspired by the one introduced by Le and Kochmann [6], but goes beyond it. In the approach used, a principal difference from the Le and Kochmann [6] model is the finite value for the initial hardening $\theta_{0}$, which becomes a key parameter for calibration. Furthermore the initial stage of the model is similar to the Kocks [16] model for dislocation density evolution and agrees with it. Thus based on calibration at any given temperature and strain rate, our model can be adjusted for any other values of strain rate and temperature causing the RX process.

As can be seen in Figure 3, the prediction of the grain size from the model gives good qualitative agreement and reasonable quantitative matching at the nanoscale, confirming an increasing grain refinement for larger plastic deformations. These results confirm that the goal of achieving nanometric scale grains is feasible as an outcome of machining.

After zero dislocation density is reached for very large plastic strains, as anticipated in section 4, other deformation mechanisms needs to be considered in order to accommodate further plastic deformations without dislocation motion. The most widely accepted mechanism consistent with this requirement is grain boundary sliding, and a new model that accounts for it is already in preparation, to be published in future work.

\section{Implementation of the model}

The proposed model is suitable for implementation in a user subroutine for any finite element (FE) software. In this section the general approach for the implementation of the model, for the prediction of the microstructural evolution, is provided. An isotropic von Mises yield criterion is considered, and a backward Euler formulation is used to compute equivalent plastic strain $\varepsilon_{p}=\gamma_{p} / \sqrt{3}$ and equivalent yield strength $\sigma_{y}=\tau_{y} \sqrt{3}$ at each step.

The function that governs the evolution of the yield surface is defined piecewise; for an equivalent plastic strain that is smaller than the critical value for $\mathrm{RX}$ initiation $\varepsilon_{p}<\varepsilon_{c}$, with 
$\varepsilon_{c}=\gamma_{c} \sqrt{3}$, eq. (9) can be used; while for larger strains $\varepsilon_{p} \geq \varepsilon_{c}$ the yield strength becomes steady state (giving perfect plasticity) and equivalent to $\sigma_{y, s s}=\tau_{y, s s} \sqrt{3}$.

Determination of the microstructural parameters can be done by solving eq. (15) numerically in order to obtain the average grain diameter $d$ from the computed $\gamma_{p}$. The dislocation density can be then obtained by substitution of that value for $d$ in eq. (14) and hence $\rho$ can be evaluated.

The implementation of this model has been done in the FE software Advant Edge (Third Wave Systems) [http://www.thirdwavesys.com/], and results for the prediction of the equivalent plastic strain, average grain size and dislocation density are shown in Figures 5-8 for the first experimental point on the left shown in Figure 3, corresponding to $\vartheta=20^{\circ}$. Coulomb friction has been considered at the interface between the chip and the cutting tool, with a coefficient of $0.5,{ }^{3}$.

In Figure 5 the distribution of the equivalent plastic strain is reported, and the average value within the chip is

$$
\left\langle\varepsilon_{p}\right\rangle=\frac{1}{\Omega_{c}} \int_{\Omega_{c}}\left(\varepsilon_{p}\right) d \Omega_{c} \approx 1,
$$

where $\Omega_{c}$ represents the chip domain. The value reported in eq. (23) gives $\left\langle\gamma_{p}\right\rangle \approx 1.7$, in agreement with the value reported on the abscissa for the first experimental point on the left in Figure 3. A thin layer that extends $\sim 50 \mu \mathrm{m}$ below the machined surface is shown to have the same average value of plastic strain as the chip (its thickness is indicated with $t$ ).

In Figure 6 the distribution of Von Mises stress is reported. In the proximity of the primary deformation zone PDZ (green and yellow shadings) the material is in the elasto-plastic regime and the Von Mises stress can reach its maximum value corresponding to the maximum yield strength given by eq. (17) (yellow shading). Far away from the PDZ the material is in elastic regime (blue and cyan shadings).

In Figure 7 the distribution of the grain size is reported; the maximum value of $d_{0}=75 \mu \mathrm{m}$ can be seen in the entire material domain where no grain refinement occurs (red shading). The average value of,

$$
\langle d\rangle=\frac{1}{\Omega_{c}} \int_{\Omega_{c}}(d) d \Omega_{c} \approx 20 \mathrm{~nm},
$$

\footnotetext{
3 In the experiments performed by Shankar et al [1] the measure of such coefficient is not reported. Its value influences the amount of plastic strain that accumulated inside the chip, therefore the value of 0.5 has been chosen in order to match the latter quantity.
} 
can be seen within the chip domain (blue shading) and is equivalent to the average value that can be seen in the thin layer that extends below the machined surface. Intermediate values, between the one reported in eq. (24) and $d_{0}$, can be seen in the primary deformation zone (PDZ) and below the thin layer.

In Figure 8 the distribution of dislocation density is reported. The maximum dislocation density corresponds to the critical density for RX initiation, and occurs at the left end of the PDZ and below the thin surface layer (red shading), corresponding to the fact that dislocations tend to accumulate until the critical density for $\mathrm{RX}$ initiation is reached, following the law reported in eq. (7). After this density is reached, continued plastic strain can only reduce the density because of dislocation absorption by new grain boundaries, as shown in Figure 4. As a result, on the right end of the PDZ, within the chip domain, and within the thin surface layer, the dislocation density is smaller than the maximum value obtained in the calculation, $\rho_{c}=\left(\tau_{y, s s} / b \mu\right)^{2} \approx 610^{14}$.

\section{Conclusions}

A thermodynamically consistent constitutive model that accounts for grain refinement by continuous dynamic recrystallization (CDRX) has been provided in this paper. This model creates a link between the energy stored by the microstructure (the latent heat) and the process of CDRX, and it predicts the feasibility of obtaining nanostructured metals with processes involving severe plastic deformation (SPD) such as cold work. Despite the simplicity of the model, a qualitative matching in the prediction of the microstructural evolution, for grain diameters at the nanoscale, is obtained.

The proposed model can be calibrated for any experiment involving cold work and for any polycrystalline metal. As discussed in this paper, for machining experiments performed at sufficiently low cutting speed, and thus involving a low strain rate, the temperature rise can be negligible and thermal softening is absent. In such cases the hypothesis of steady-state yield strength during CDRX is valid. Thus the model presented in this paper can be used in simulations of processes involving SPD if the strain-rates are low enough to avoid thermal softening.

Acknowledgments - The support by the Department of Energy and Third Wave Systems, under grant DE-FG03-99ER-45768 is greatly appreciated.

Disclaimer - This report was prepared as an account of work sponsored by an agency of the United States Government. Neither the United States Government nor any agency thereof, nor any of their employees, makes any warranty, express or implied, or assumes any legal liability or responsibility for the accuracy, completeness, or usefulness of any information, apparatus, product, or process disclosed, or represents that its use would not infringe privately owned rights. Reference herein to any specific commercial product, process, or service by trade name, trademark, manufacturer, or otherwise does not necessarily constitute or imply its endorsement, recommendation, or favoring by the United States Government or any agency thereof. The views and opinions of authors expressed herein do not necessarily state or reflect those of the United States Government or any agency thereof. 


\section{References}

1. M. R. Shankar, S. Chandrasekar, A. H. King, W. D. Compton (2005) "Microstructure and stability of nanocrystalline aluminum 6061 created by large strain machining", Acta Mater 53 pp. 4781-4793.

2. A. Mishra, V. Richard, F. Grégori, R.J. Asaro, M.A. Meyers (2005) "Microstructural evolution in copper processed by severe plastic deformation" Mat. Sci. Eng. A 410 pp. 290-298.

3. R.Z. Valiev, T.G. Langdon (2006) "Principles of equal-channel angular pressing as a processing tool for grain refinement", Prog. Mater. Sci. 51 pp. 881-981.

4. E.O. Hall (1951) "The deformation and ageing of mild steel", Proc. Phys. Soc. B64 pp. 742-753.

5. N.J. Petch (1953) "The cleavage strength of polycrystals", J.Iron Steel Inst. 174 pp. 2528.

6. K.C. Le, D.M. Kochmann (2009) "A simple model for dynamic recrystallization during severe plastic deformation", Arch. Appl. Mech. 79 pp. 579-586.

7. J.R. Rice (1971). "Inelastic constitutive relations for solids: An internal-variable theory and its application to metal plasticity", J. Mech. Phys. Solids 19 pp. 433-455.

8. G.I. Taylor (1934) "The mechanism of plastic deformation of crystals", Proc.Roy.Soc.Lond. A145 pp. 362-387.

9. F.R. Castro-Fernandez, C.M. Sellars (1989) "Relationship between room-temperature proof stress, dislocation density and subgrain size", Phil. Mag. A 60(4) pp. 487-506.

10. G.I. Taylor, H. Quinney (1934) "The latent energy remaining in a metal after cold working", Proc.R.Soc.A 143 pp. 307-326.

11. V.L. Berdichevsky (2006) "On thermodynamics of crystal plasticity", Scr.Mater. 54 pp. 711-716.

12. W. Roberts, B. Ahlblom, (1978) "A nucleation criterion for dynamic recrystallization during hot working", Acta Metall. 26(5) pp. 801-813.

13. J. E. Bailey and P. B. Hirsch (1961) "The Recrystallization Process in Some Polycrystalline Metals". Proc. R. Soc. A 267 pp. 11-30.

14. M. J. Luton, C. M. Sellars (1969) "Dynamic recrystallization in nickel and nickel-iron alloys during high temperature deformation", Acta Metall. 17(8) pp. 1033-1043.

15. M.E. Kassner, S.R. Barrabes (2005) "New developments in geometric dynamic recrystallization", Mat. Sci. Eng. A 140(411) pp. 152-155.

16. U. F. Kocks (1976) "Laws for Work-Hardening and Low-Temperature Creep", J. Eng Mater. Tech. (ASME H) 98 pp. 76-85.

17. E. Voce (1948) "The Relationship between Stress and Strain for Homogeneous Deformation", J. Inst. Metals 74 pp. 537-562.

18. G. Nurislamova, X. Sauvage, M. Murashkin, R. Islamgaliev, R. Valiev (2008) "Nanostructure and related mechanical properties of an Al-Mg-Si alloy processed by severe plastic deformation", Philos. Mag. Lett. 88(6) pp. 459-466.

19. S. Gourdet, F. Motheillet (2003) "A model of continuous dynamic recrystallization", Acta Mater. 51 pp. 2685-2699.

20. A. A. Brown, D. J. Bammann (2012) "Validation of a model for static and dynamic recrystallization in metals", Int. J. Plasticity 32-33 pp. 17-35. 


\section{Figures}

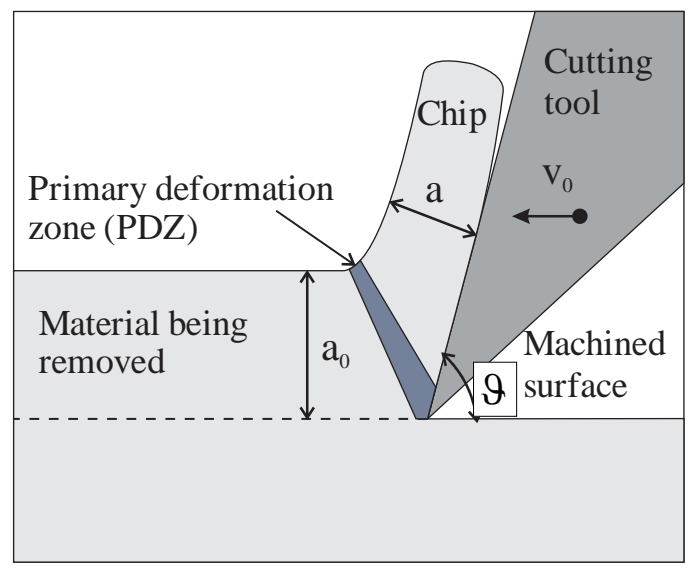

Figure 1: Schematic representation of the machining experiment used for the validation of the proposed model. In this picture, the key parameters for machining are shown and their value for the experiment of Shankar et. al. [1] are reported in Table 2. 


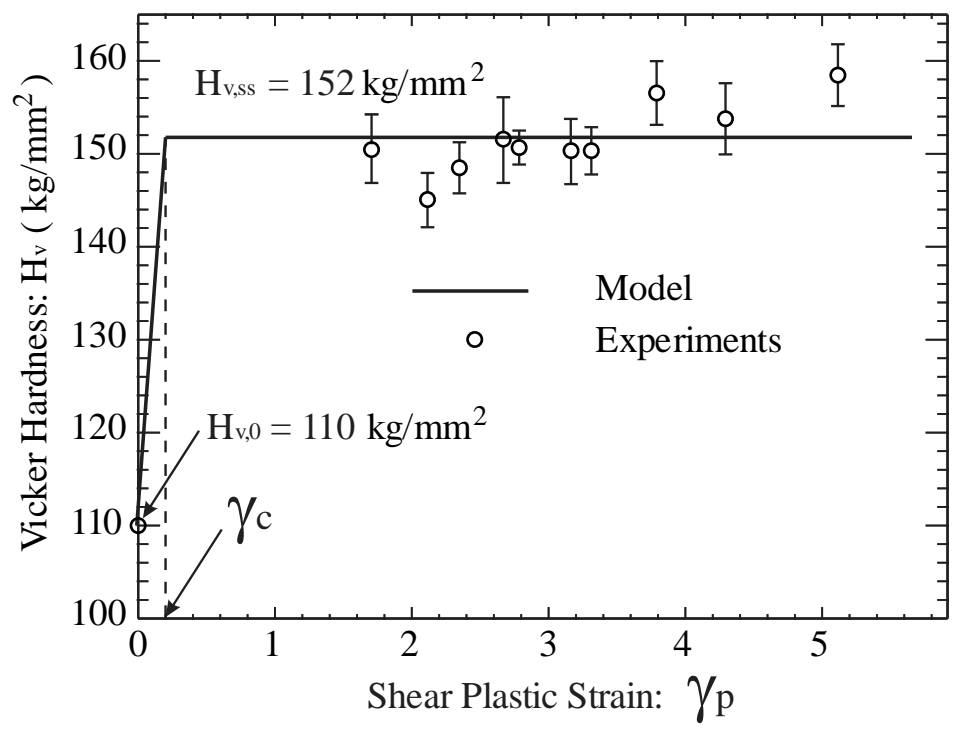

Figure 2: Variation of Vicker hardness $H_{v}$ with shear equivalent plastic strain $\gamma_{p}$ for chips cut from peak-aged aluminum 6061. Hardness of peak-aged alloy prior to machining is $110 \mathrm{Kg} / \mathrm{mm}^{2}$. Vickers indentation load: $50 \mathrm{~g}$ [1]. 


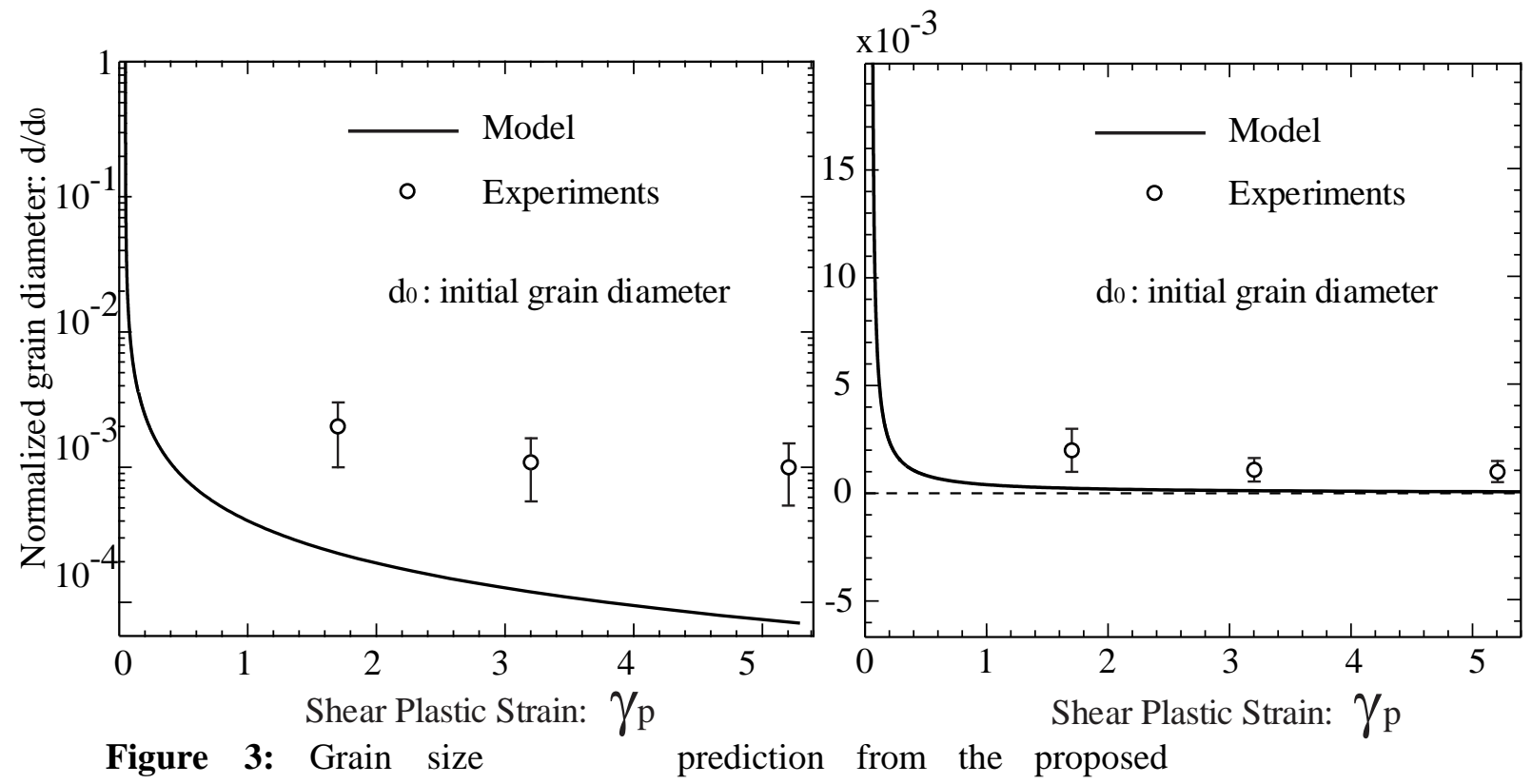

model (solid line) and its comparison with experiments on machining (open circles with error bars) [1]. On the left is a logarithmic plot of the normalized grain diameter $d / d_{0}$ whereas on the right is an arithmetic plot. 


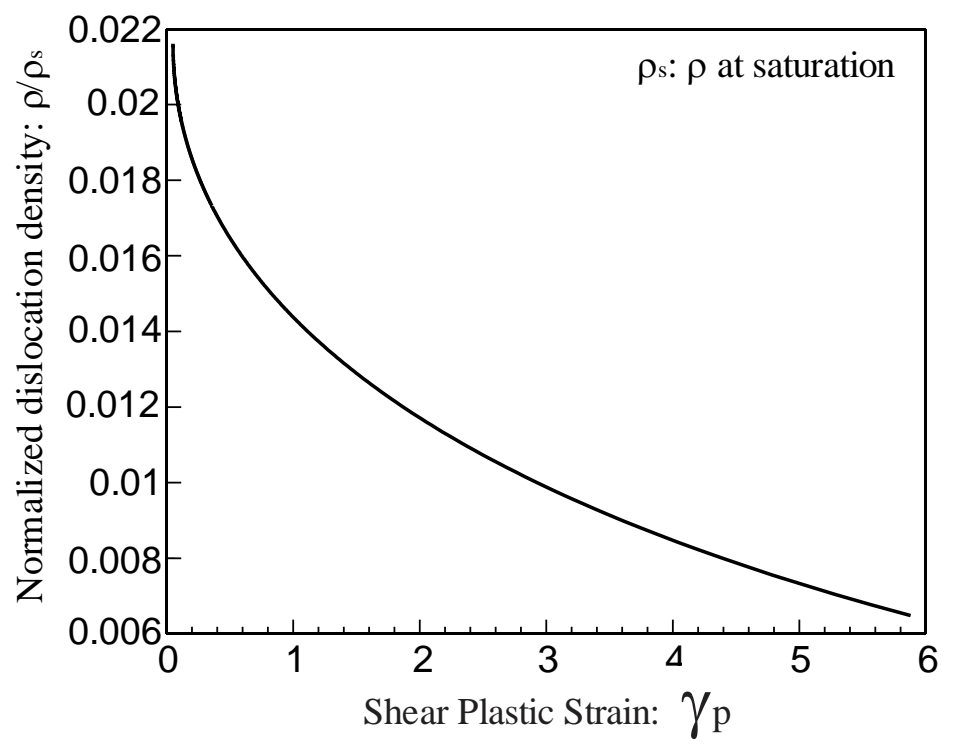

Figure 4 Normalized dislocation density evolution during continuous dynamic recrystallization (CDRX) in the proposed model. 


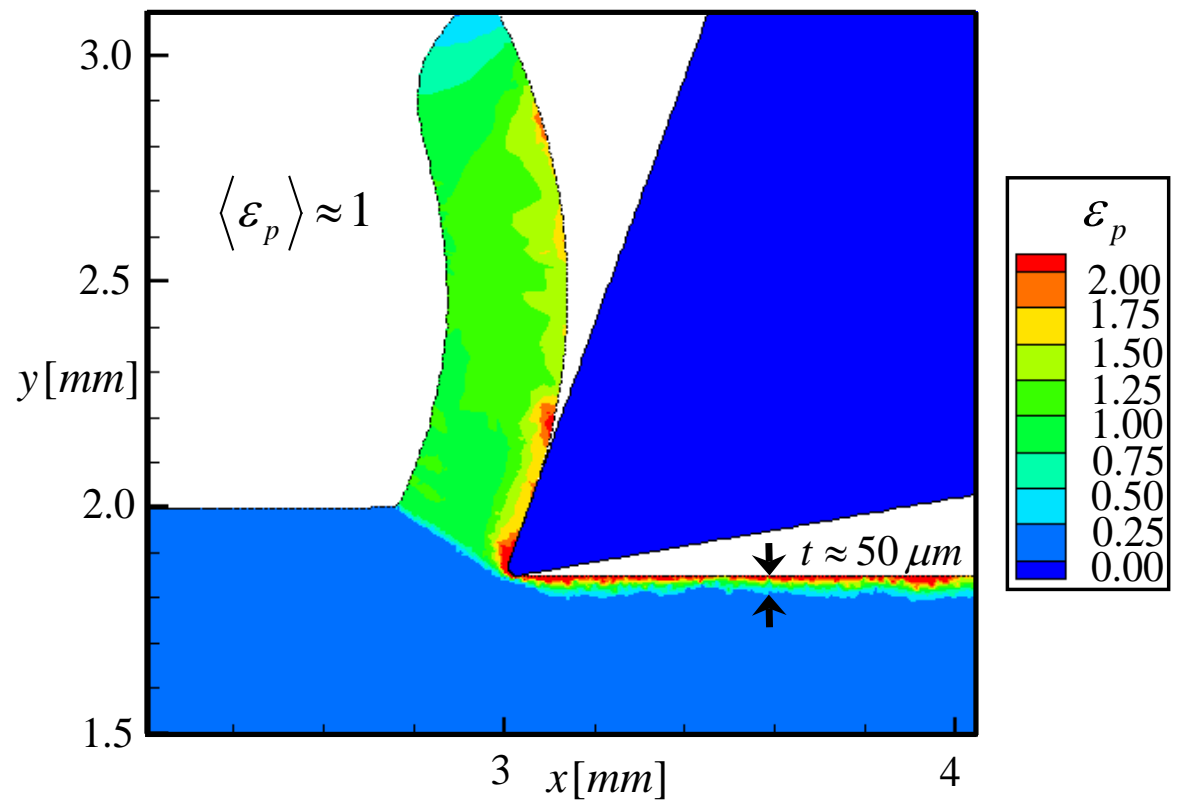

Figure 5 Distribution of equivalent plastic strain for a machining simulation with low cutting speed $v_{c} \approx 10 \mathrm{~mm} / \mathrm{s}$, rake angle $20^{\circ}$, depth of cut $a_{0}=150 \mu \mathrm{m}$, (from experiments [1]), which gives $\left\langle\varepsilon_{p}\right\rangle=1$ as average values within the chip domain, so that $\left\langle\gamma_{p}\right\rangle=1.7$ in agreement with the first experimental point in Figure 3. 


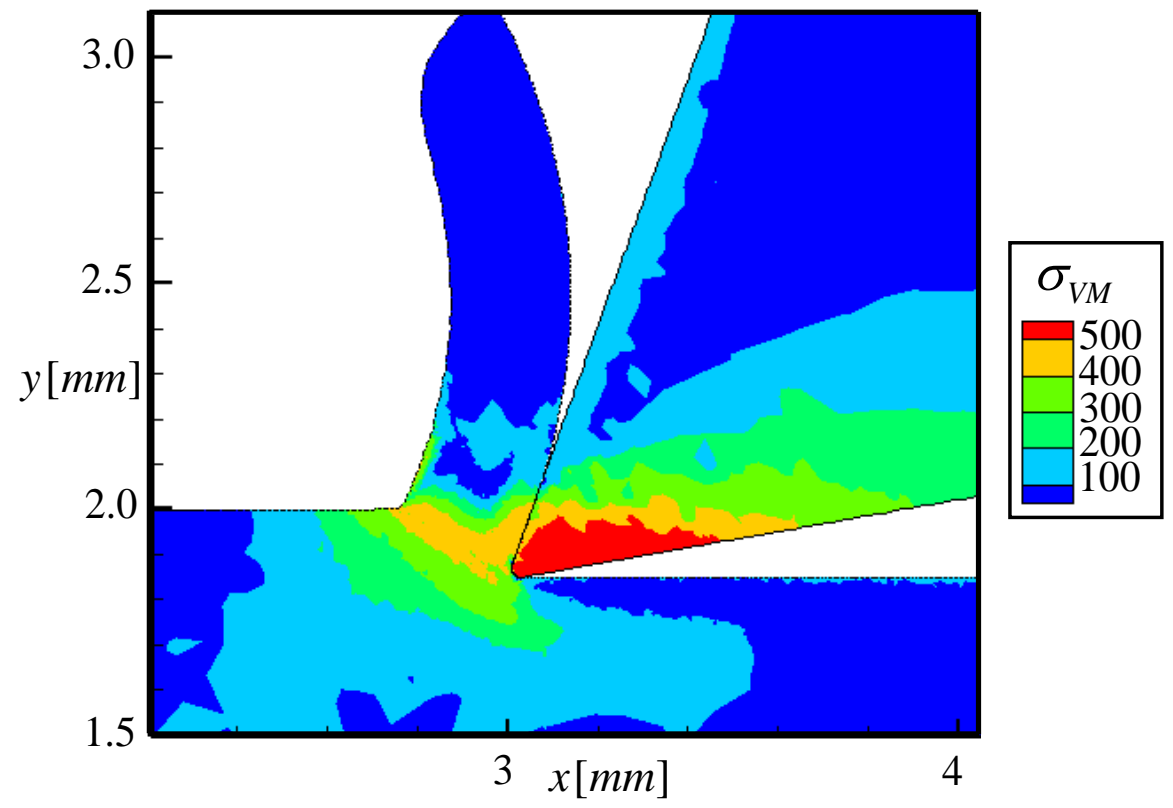

Figure 6 Distribution of the Von Mises stress for a machining simulation with the same conditions as Figure 5. In the proximity of the primary deformation zone PDZ (green and yellow shadings) the material is in the elasto-plastic regime and the Von Mises stress reaches its maximum value that corresponds to the maximum yield strength given by eq. (17). Far away from the PDZ the material is in elastic regime (blue and cyan shadings). 


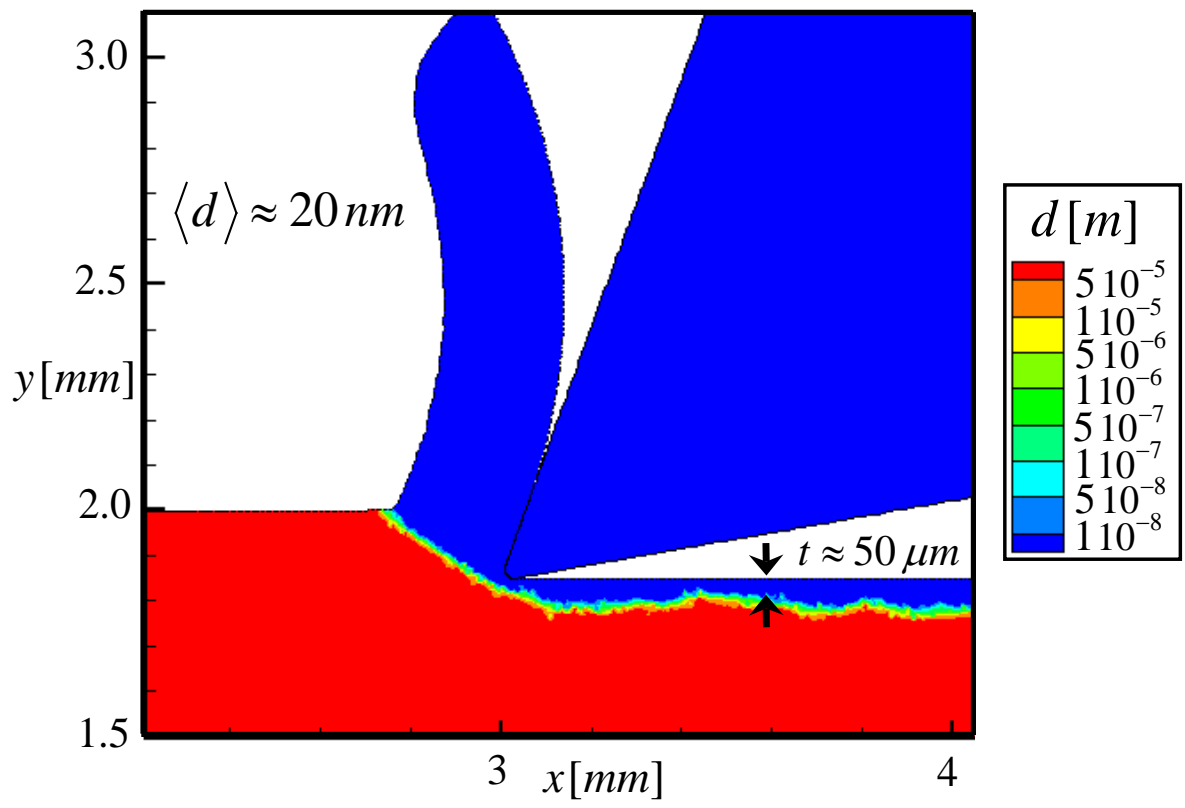

Figure 7 Distribution of average grain diameter for a machining simulation with the same conditions as Figure 5. The results can be compared with the first experimental point in Figure 3. As showed in both Figure 3 and this figure, the average grain size is $\langle d\rangle \approx 20 \mathrm{~nm}$, as reported in eq. (24), with the ratio $\langle d\rangle / d_{0} \approx 2.710^{-4}$. 


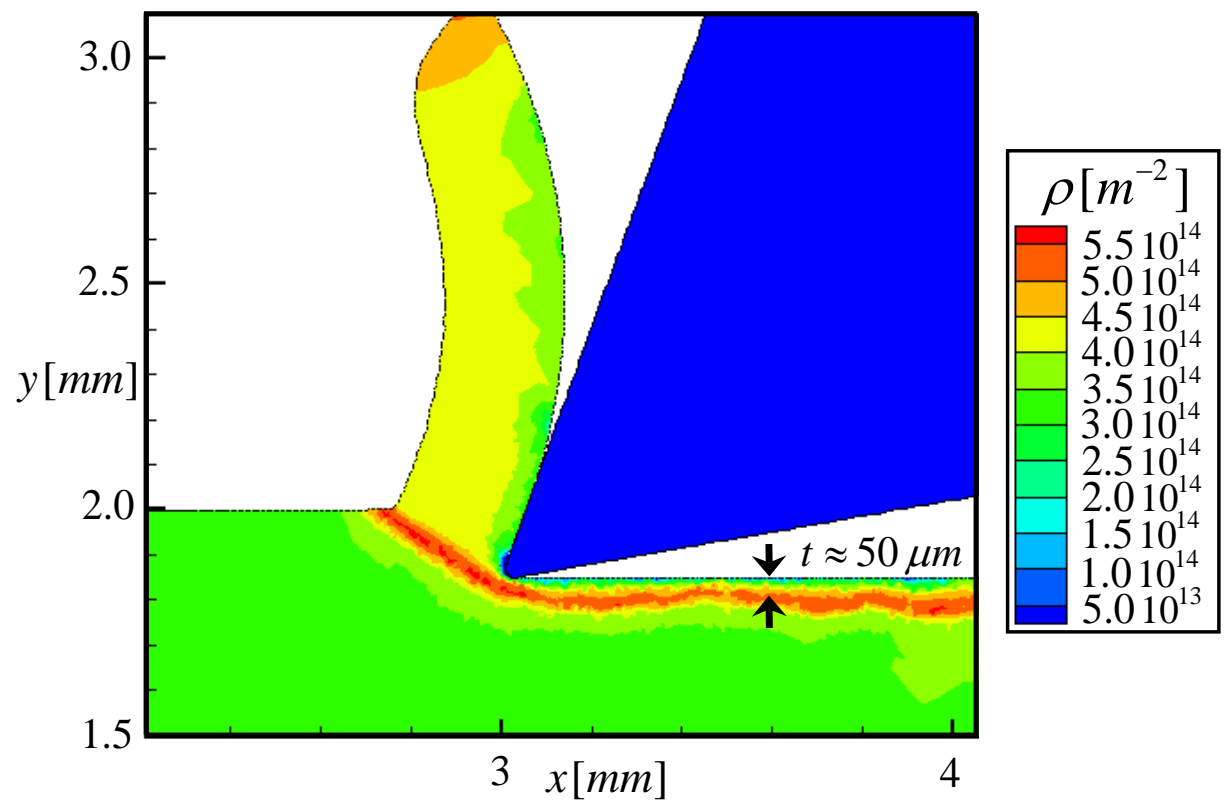

Figure 8 Distribution of dislocation density for a machining simulation with the same conditions as Figure 5. The results can be compared to Figure 4 with a shear plastic strain of $\gamma_{p}=1.7$. Maximum amount of dislocation density is reached before recrystallization starts (red shading) and it corresponds to $\rho_{c}=\left(\tau_{y, s s} / b \mu\right)^{2} \approx 610^{14}$. 